\title{
LOCALLY FINITE FAMILIES, COMPLETELY SEPARATED SETS AND REMOTE POINTS
}

\author{
M. HENRIKSEN AND T. J. PETERS
}

(Communicated by Doug W. Curtis)

\begin{abstract}
It is shown that if $X$ is a nonpseudocompact space with a $\sigma$ locally finite $\pi$-base, then $X$ has remote points. Within the class of spaces possessing a $\sigma$-locally finite $\pi$-base, this result extends the work of Chae and Smith, because their work utilized normality to achieve complete separation. It provides spaces which have remote points, where the spaces do not satisfy the conditions required in the previous works by Dow, by van Douwen, by van Mill, or by Peters.

The lemma: "Let $X$ be a space and let $\left\{C_{\xi}: \xi<\alpha\right\}$ be a locally finite family of cozero sets of $X$. Let $\left\{Z_{\xi}: \xi<\alpha\right\}$ be a family of zero sets of $X$ such that for each $\xi<\alpha, Z_{\xi} \subset C_{\xi}$. Then $\bigcup_{\xi<\alpha} Z_{\xi}$ is completely separated from $X / \bigcup_{\xi<\alpha} C_{\xi}$ ", is a fundamental tool in this work.

An example is given which demonstrates the value of this tool. The example also refutes an appealing conjecture-a conjecture for which the authors found that there existed significant confusion within the topological community as to its truth or falsity.
\end{abstract}

1. Introduction. All spaces that appear below are assumed to be Tychonoff. A $\pi$-base for a space $X$ is a collection of open subsets such that each open subset of $X$ contains one of them. A space that has a $\sigma$-locally finite $\pi$-base is called a $\sigma-\pi$ space. As usual, $\beta X$ will denote the Stone-Cech compactification of $X$, and a point $p$ of $\beta X$ that fails to be in $\operatorname{cl}_{\beta X} A$ for any nowhere dense subset $A$ of $X$ is called a remote point of $X$, and $T X$ will denote the set of remote points of $X$. The cardinality of $X$ will be denoted by $|X|$.

The existence of remote points for large classes was established first by N. Fine and L. Gillman in [FG] assuming the continuum hypothesis, and this assumption was shown to be unnecessary by E. van Douwen and S. Chae and J. Smith [VD, CS]. In particular, they show that every space with countable $\pi$-weight has a remote point.

Recall from [CS] that a space $X$ is called a $G$-space if for any open subspace $U$ of $X$ and any positive integer $n$, there is a family of $\mathbf{C}$ of nonempty closed sets with the $n$-intersection property such that every dense open set in $U$ contains a set in C.

They showed that every normal nonpseudocompact $G$-space has remote points and that every $\sigma-\pi$ space is a $G$-space. In particular, every metrizable space is a $G$-space. However, there exist $G$-spaces which are not $\sigma$ - $\pi$ spaces $\left[\mathbf{P}_{\mathbf{2}}, \mathbf{P}_{\mathbf{4}}\right]$. In this work, it is shown that every nonpseudocompact $\sigma-\pi$ space has remote points.

Received by the editors May 14, 1986 and, in revised form, April 2, 1987.

1980 Mathematics Subject Classification (1985 Revision). Primary 54D40, 54D35, 54B10, 03E35, 03E55; Secondary 54A25, 54B25, 54G20. 
The question of the existence of remote points for nonnormal, nonpseudocompact $G$-spaces, as posed in $\left[\mathbf{P}_{\mathbf{2}}, \mathbf{P}_{\mathbf{3}}\right]$, remains open.

2. Complete separation via locally finite families. Pervading the literature on remote points has been a concern with techniques for the demonstration of cornplete separation [CS, vD, D, FG, G, vM, $\left.\mathbf{P}_{\mathbf{1}}, \mathbf{P}_{\mathbf{2}}, \mathbf{P}_{\mathbf{3}}, \mathbf{R}, \mathbf{W}\right]$. This work shares that concern, as evidenced by the following lemma.

2.1 LEMMA. Let $X$ be a space and let $\left\{C_{\xi}: \xi<\alpha\right\}$ be a locally finite family of cozero sets of $X$. Let $\left\{Z_{\xi}: \xi<\alpha\right\}$ be a family of zero sets of $X$ such that for each $\xi<\alpha, Z_{\xi} \subseteq C_{\xi}$. Then $\bigcup_{\xi<\alpha} Z_{\xi}$ is completely separated from $X \backslash \bigcup_{\xi<\alpha} C_{\xi}$. that

Proof. For each $\xi<\alpha$, there exists a continuous function $f_{\xi}: X \rightarrow[0,1]$ such

$$
\begin{array}{ll}
f_{\xi}(x)=1 & \text { for all } x \in Z_{\xi} \quad \text { and } \\
f_{\xi}(x)=0 & \text { for all } x \in X \backslash C_{\xi} .
\end{array}
$$

Define the function $f: X \rightarrow[0, \infty)$ by $f=\sum_{\xi<\alpha} f_{\xi}$. The local finiteness of $\left\{C_{\xi}: \xi<\alpha\right\}$ insures that $f$ is well defined and continuous.

Furthermore,

$$
\begin{aligned}
& f(x) \geq 1 \quad \text { for all } x \in \bigcup_{\xi<\alpha} Z_{\xi} \quad \text { and } \\
& f(x)=0 \quad \text { for all } x \in X / \bigcup_{\xi<\alpha} C_{\xi}
\end{aligned}
$$

which suffices to prove the lemma [GJ, 1.15].

3. Remote points for $\sigma-\pi$ spaces. The appropriate modifications are now made to the techniques of Chae and Smith so that the previous lemma may be invoked in lieu of their hypothesis of normality.

3.1 THEOREM. If $X$ is a nonspeudocompact $\sigma-\pi$ space, then $|T X| \geq 2^{c}$.

ProOF. Let $\mathbf{B}=\bigcup_{n<\omega} \mathbf{B}_{n}$ be a $\sigma$-locally $\pi$-base for $X$. Without loss of generality, assume that for each $n<\omega, \mathbf{B}_{n} \subset \mathbf{B}_{n+1}$, and further, assume that each $B \in \mathbf{B}$ is a cozero set of $X$. Let $U$ be a nonempty open subset of $X$. Then there exists $n<\omega$ and $V \in \mathbf{B}_{n}$ such that $V \subset U$. Let $\#(U)$ be the least such $n$. Furthermore, there exists $m<\omega$ and $W \in \mathbf{B}_{m}$ such that $W$ is completely separated from $X \backslash V$

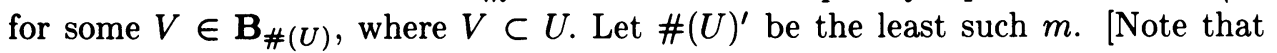
$\#(U)^{\prime} \geq \#(U)$.]

Let

$$
\begin{array}{r}
\tilde{\mathbf{B}}_{\#(U)}=\left\{V \in \mathbf{B}_{\#(U)}: V \subset U \text { and there exists } W \in \mathbf{B}_{\#(U)^{\prime}}\right. \text { such that } \\
W \text { is completely separated from } X \backslash V\} .
\end{array}
$$

For each $V \in \tilde{\mathbf{B}}_{\#(U)}$, choose a unique $W_{V} \in \mathbf{B}_{\#(U)^{\prime}}$ such that $W_{V}$ is completely separated from $X \backslash V$ and also choose a unique zero set $Z_{W_{V}}$ such that $W_{V} \subset$ $Z_{W_{V}} \subset V$. The unique choices insure that the family $\left\{Z_{W_{V}}: V \in \tilde{\mathbf{B}}_{\#(U)}\right\}$ is locally finite.

Let

$$
F_{1}(U)=\bigcup\left\{Z_{W_{V}}: V \in \tilde{\mathbf{B}}_{\#(U)}\right\}
$$


Inductively, for each $n<\omega$, define

$$
F_{n}(U)=\bigcup\left\{F_{n-1}(U \cap W): W \in \mathbf{B}_{\#(U)^{\prime}} \text { and } U \cap W \neq \varnothing\right\} \cup F_{n-1}(U) .
$$

It is clear that each $F_{n}(U)$ is closed and each $F_{n}(U) \subset U$.

Let $\mathbf{H}$ be the family of dense open subsets of $X$. For each $n<\omega$ and for each nonempty open subset $U$ of $X$, let

$$
\mathbf{F}_{n}(U)=\left\{F_{n}(U \cap H): H \in \mathbf{H}\right\} .
$$

The argument of [CS, Theorem 3] is now adapted to show that $\mathbf{F}_{n}(U)$ has the $n$-intersection property. The argument proceeds by induction.

Let $H_{1}, H_{2}, \ldots, H_{n} \in \mathbf{H}$.

For $n=1$, it is clear that $F_{1}\left(U \cap H_{1}\right)$ is nonempty.

Suppose that the statement is true for $n-1$. Without loss of generality, assume that \# $\left(U \cap H_{1}\right)^{\prime}$ is less than \# $\left(U \cap H_{i}\right)^{\prime}$, for $i=2, \ldots, n$. (Hence, $\mathbf{B}_{\#\left(U \cap H_{1}\right)^{\prime}} \subset$ $\mathbf{B}_{\#\left(U \cap H_{i}\right)^{\prime}}$, for $i=2, \ldots, n$.) By definition of $F_{n}(\cdot)$, there exist $V \in \mathbf{B}_{\#\left(U \cap H_{1}\right)}$, $W_{V} \in \mathbf{B}_{\#\left(U \cap H_{1}\right)^{\prime}}$ and $Z_{W_{V}}$ such that $W_{V} \subset Z_{W_{V}} \subset F_{1}\left(U \cap H_{1}\right) \subset F_{n}\left(U \cap H_{1}\right) \subset$ $U \cap H_{1}$. Hence, $U \cap W_{V}$ is a nonempty open subset of $X$.

Let

$$
L=F_{n-1}\left(U \cap W_{V} \cap H_{2}\right) \cap \cdots \cap F_{n-1}\left(U \cap W_{V} \cap H_{n}\right) .
$$

The induction hypothesis implies that $L$ is nonempty. Since $W_{V} \in \mathbf{B}_{\#\left(U \cap H_{i}\right)^{\prime}}$ whenever $i=2, \ldots, n$, it follows that $L \subset F_{n-1}\left(U \cap W_{V} \cap H_{i}\right) \subset F_{n}\left(U \cap H_{i}\right)$ for $i=$ $2, \ldots, n$-where the latter inclusion follows from the definition of $F_{n}(\cdot)$. Note also that $L \subset F_{n-1}\left(U \cap W_{V} \cap H_{2}\right) \subset W_{V} \subset F_{n}\left(U \cap H_{1}\right)$. Hence, $L \subset F_{n}\left(U \cap H_{1}\right) \cap \cdots \cap F_{n}\left(U \cap H_{n}\right)$. Therefore, the family $\mathbf{F}_{n}(U)$ has the $n$ intersection property.

Let $\left\{G_{n}: n<\omega\right\}$ be a countably infinite discrete family of nonempty cozero sets of $X$. For each $q \in \omega^{*}$, let

$$
\mathbf{F}_{q}=\left\{\bigcup_{n \in J} F_{n}\left(G_{n} \cap H\right): J \in q, H \in \mathbf{H}\right\} .
$$

It is easy to see that $\bigcap \mathbf{F}_{q}=\varnothing$ and that $\mathbf{F}_{q}$ is a family of closed subsets of $X$, where $\mathbf{F}_{q}$ has the finite intersection property. So, for each $q \in \omega^{*}$, it follows that

$$
\bigcap\left\{\operatorname{cl}_{\beta X} F: F \in \mathbf{F}_{q}\right\} \subset \beta X / X \text {. }
$$

It remains to show, for each $q \in \omega^{*}$, that $\bigcap\left\{\operatorname{cl}_{\beta X} F: F \in \mathbf{F}_{q}\right\} \subset T X$. Clearly, it suffices for each such $q$ and each nowhere dense $A \subset X$ to show that there exists $F_{A} \in \mathbf{F}_{q}$ such that $F_{A}$ is completely separated from $A$. Let $H \in \mathbf{H}$ such that $A \subset X \backslash H$. Let $F_{A}=\bigcup_{n<\omega} F_{n}\left(G_{n} \cap H\right)$. That $A$ and $F_{A}$ are completely separated can be seen by invoking Lemma 2.1 .

It now only remains to show that the cardinality of $T X$ is at least $2^{c}$. The argument here is an adaptation of [vD]. For each $q \in \omega^{*}$, let

$$
T_{q}=\bigcap\left\{\operatorname{cl}_{\beta X} F: F \in \mathbf{F}_{q}\right\} .
$$

Let $H \in \mathbf{H}$, let $p, q \in \omega^{*}$ such that $p \neq q$ and let $J \in p, K \in q$ such that $J$ and $K$ are disjoint. Then

$$
T_{p} \subset \operatorname{cl}_{\beta X} \bigcup_{n \in J} F_{n}\left(G_{n} \cap H\right) \quad \text { and } \quad T_{q}=\operatorname{cl}_{\beta X} \bigcup_{n \in K} F_{n}\left(G_{n} \cap H\right) .
$$


Note that the condition that for each $n<\omega, G_{n}$ is a cozero set, implies that $X \backslash \bigcup_{n \in J} G_{n}$ is a zero set. Furthermore,

$$
\bigcup_{n \in K} F_{n}\left(G_{n} \cap H\right) \subset X \backslash \bigcup_{n \in J} G_{n} \text { and } \bigcup_{n \in J} F_{n}\left(G_{n} \cap H\right) \subset \bigcup_{n \in J} G_{n} .
$$

Then, the definition of $F(\cdot)$, the discreteness of the family $\left\{G_{n}: n<\omega\right\}$ and Lemma 2.1 insure that $\bigcup_{n \in J} F_{n}\left(G_{n} \cap H\right)$ is completely separated from $\bigcup_{n \in K} F_{n}\left(G_{n} \cap H\right)$. Hence, $T_{p}$ and $T_{q}$ are disjoint. Since the cardinality of $\omega^{*}$ is equal to $2^{c}$, it is clear that the cardinality of $T X$ is at least $2^{c}$, and the proof is complete.

3.2 REMARK. Many examples of $\sigma-\pi$ products are given in $\left[\mathbf{P}_{\mathbf{1}}\right.$ and $\left.\mathbf{P}_{\mathbf{4}}\right]$. Certain specialized instances of these products had been shown to have remote points $\left[\mathbf{P}_{\mathbf{1}}, \mathbf{P}_{\mathbf{3}}\right]$. Theorem 3.1 is much more general.

In particular, Theorem 3.1 demonstrates that the following product has remote points - a result that was previously unknown.

3.3 EXAMPLE. Let $\left\{X_{\xi}\right\}_{\xi<\omega_{1}}$ be the family of discrete spaces, where $X_{\xi}=\omega_{\xi+1}$, for each $\xi<\omega_{1}$. Since the product $\prod_{\xi<\omega_{1}} X_{\xi}$ is a $\sigma-\pi$ space $\left[\mathbf{P}_{1}, \mathbf{P}_{4}\right]$, it also has remote points.

4. The class of $\sigma-\pi$ spaces versus other classes of spaces known to possess remote points. It is worthwhile to note the difference between the class of $\sigma-\pi$ spaces and some other similar classes of spaces known to possess remote points.

A. Dow [D] showed that any nonpseudocompact space with a "...good $\pi$ base..." has remote points. The ensuing arguments demonstrate that there exist nonpseudocompact $\sigma-\pi$ spaces which do not have good $\pi$-bases.

4.1 Definition. If $\mathfrak{A}, \mathfrak{B}, \mathfrak{C} \subset \mathscr{P}(X)$, we shall say that $\mathfrak{C}$ refines $\mathfrak{A} \cap \mathfrak{B}$ if for $a \in \mathfrak{A}, b \in \mathfrak{B}$ with $a \cap b \neq \varnothing$, there is a $c \in \mathfrak{C}$ with $\bar{c} \subset a \cap b[\mathbf{D}]$.

4.2 Definition. A set $\mathfrak{A} \subset \mathscr{P}(X)$ is wide if for any maximal family of open sets, $\sigma$, there is a finite $\sigma_{1} \subset \sigma$ such that $\bigcup \sigma_{1} \cap A \neq \varnothing$ for all $A \in \mathfrak{A}$. And $\mathfrak{A}=\bigcup_{m \in \omega} \mathfrak{A}_{m} \subseteq \mathscr{P}(X)$ is good if each $\mathfrak{A}_{m}$ is wide, $\mathfrak{A}_{m} \subset \mathfrak{A}_{m+1}$, and for any $n, m \in \omega$, there is a $k \in \omega$ such that $\mathfrak{A}_{k}$ refines $\mathfrak{A}_{m} \cap \mathfrak{A}_{n}[\mathbf{D}]$.

For the following, if $X$ is a space, let $c X$ denote the cellularity of $X$.

4.3 LEMMA. If a space $X$ has a good $\pi$-base, then $X$ has the countable chain condition.

Proof. Suppose $c X$ is uncountable, and let $\mathbf{B}=\bigcup_{m<\omega} \mathbf{B}_{m}$ be a good $\pi$-base for $X$. Let $\mathfrak{C}$ be an uncountable maximal cellular family of nonvoid open subsets of $X$. For each $n<\omega$, there exists $\mathfrak{C}_{m}^{\prime} \subset \mathfrak{C}$ such that $\left|\mathfrak{C}_{m}^{\prime}\right|<\omega$ and such that $\left(\bigcup \mathfrak{C}_{m}^{\prime}\right) \cap B \neq \varnothing$, for each $B \in \mathbf{B}_{m}$. Note that $\left|\bigcup_{m<\omega} \mathfrak{C}_{m}^{\prime}\right| \leq \omega$.

Choose $\hat{C} \in \mathfrak{C}$ such that $\hat{C} \notin \bigcup_{m<\omega} \mathfrak{C}_{m}^{\prime}$.

Choose $\hat{B} \in \mathbf{B}$ such that $\hat{B} \subset \hat{C}$, where $\hat{B} \in B_{\hat{n}}$, for some $\hat{n}<\omega$.

However, then $\hat{B} \cap\left(\bigcup \mathfrak{C}_{\hat{m}}^{\prime}\right)=\varnothing$, in contradiction to the condition that $X$ has a good $\pi$-base.

4.4 REMARK. Lemma 4.3 demonstrates that any $\sigma-\pi$ space with uncountable cellularity does not have a good $\pi$-base. In particular, the product given in Example 3.3 is such a space. 
J. van Mill showed that each nonspeudocompact, “... nicely approximated spaced..." has remote points $[\mathbf{v M}]$. Remark 4.9 below shows that it is easy to find $\sigma-\pi$ spaces which are not nicely approximated.

4.5 DEFINITION. The notation $\left(X_{\alpha}, f_{\alpha \beta}, \kappa\right)$ means that $\kappa$ is an ordinal, that for each $\alpha<\kappa, X_{\alpha}$ is a space and that for each $\beta<\alpha, f_{\alpha \beta}$ is a continuous function from $X_{\alpha}$ into $X_{\beta}$ such that if $\beta<\alpha<\gamma$ then $f_{\gamma \beta}=f_{\alpha \beta} \circ f_{\gamma \alpha}$. The triple $\left(X_{\alpha}, f_{\alpha \beta}, \kappa\right)$ is called an inverse system. The inverse limit $\lim \left(X_{\alpha}, f_{\alpha \beta}, \kappa\right)$ of the inverse system $\left(X_{\alpha}, f_{\alpha \beta}, \kappa\right)$ is the subspace $\left\{x \in \prod_{\alpha<\kappa} X_{\alpha} \overleftarrow{\forall \beta}<\alpha<\kappa: X_{\beta}=f_{\alpha \beta}\left(X_{\alpha}\right)\right\}$ of $\prod_{\alpha<\kappa} X_{\alpha}$. The projection from $\lim _{(}\left(X_{\alpha}, f_{\alpha \beta}, \kappa\right)$ into $X_{\alpha}$ is denoted by $f_{\kappa \alpha}$. An inverse system $\left(X_{\alpha}, f_{\alpha \beta}, \kappa\right)$ is called continuous provided that $X_{\beta}=\underset{\lim }{\longleftarrow}\left(X_{\alpha}, f_{\alpha \gamma}, \beta\right)$ for each limit ordinal $\beta<\kappa[\mathbf{v M}]$.

4.6 DEFINITION. A continuous function $f: X \rightarrow Y$ is called quasi-open whenever $\operatorname{int}_{Y} F[U] \neq 0$ for each nonempty open $U \subset X[\mathbf{v M}]$.

4.7 DEFINITION. A space $X$ is called nicely approximated provided there is a continuous inverse system $\left(X_{\alpha}, f_{\alpha \beta}, \omega_{1}\right)$ such that

- each $f_{\alpha \beta}$ is quasi-open $\left(\beta<\alpha<\omega_{1}\right)$;

-each $X_{\alpha}$ has countable $\pi$-weight;

$-X \approx \lim _{\alpha}\left(X_{\alpha}, f_{\alpha \beta}, \omega_{1}\right)$.

We say that $\left(X_{\alpha}, f_{\alpha \beta}, \omega_{1}\right)$ nicely approximates $X[\mathbf{v M}]$.

4.8 LEMMA. If $X$ is a nicely approximated space, then $|X| \leq 2^{c}$.

PROOF. it is clear that $X$ is homeomorphic to a subspace of a product of $\omega_{1}$ many factors, where each factor has countable $\pi$-weight. Hence, the full product is separable $[\mathbf{C}]$. Therefore, the cardinality of the full product must be less than or equal to $2^{c}[\mathbf{C}]$. Hence $|X| \leq 2^{c}$.

4.9 REMARK. Any discrete space of cardinality exceeding $2^{c}$ is a $\sigma-\pi$ space, which is not nicely approximated. It is also clear that any such spaces does not have a good $\pi$-base (4.3).

EXAMPLE 3.3 and Remark 4.9 can now be combined to give an example of a nontrivial $\sigma-\pi$ space, which neither has a good $\pi$-base nor is nicely approximated.

4.10 EXAMPLE. Let $X$ be the product given in Example 3.3. Let $D$ be the discrete space of cardinality $\left(2^{c}\right)^{+}$.

Let $Y=X \times D$. Then $Y$ is a $\sigma-\pi$ space (since $D$ is discrete and $X$ is a $\sigma-\pi$ space), but $Y$ does not have a good $\pi$-base (4.3) and $Y$ is not nicely approximated (4.8).

The work of Dow [D] and of van Mill [vM] include spaces which are not $\sigma-\pi$ spaces.

4.11 EXAMPLE. Let $X=\omega^{\omega_{1}}$, where each factor $\omega$ has the discrete topology. Then $X$ has a good $\pi$-base [D] and $X$ is nicely approximated [vM], but $X$ is not a $\sigma-\pi$ space $\left[\mathbf{P}_{\mathbf{1}}, \mathbf{P}_{\mathbf{4}}\right]$.

5. Counterexamples to conjectures concerning complete separation. Some care must be exercised in the proof of Theorem 3.1 in order to effect the desired complete separation. Naive attempts to modify the hypotheses of Lemma 2.1 and/or the proof of Theorem 3.1, easily become misdirected. In particular, negative answers are provided to two questions concerning potential avenues of modification. 
5.1 QUESTION. If $\left\{C_{\xi}: \xi<\alpha\right\}$ is a locally finite family of cozero sets of a space $X$ and if $A \subset X$ such that for each $\xi<\alpha, C_{\xi}$ and $A$ are completely separated, then must $\bigcup_{\xi<\alpha} C_{\xi}$ and $A$ be completely separated? (The answer is "No.", as is demonstrated by Example 5.2, below.)

5.2 EXAMPLE. Let $\omega$ have the discrete topology and let $(\omega+1)$ be its one-point compactification.

Let $\tilde{\omega}_{1}$ consist of the space $\omega_{1}+1$ endowed with the following topology. If $\alpha<\omega_{1}$, then $\{\alpha\}$ is open. If $U \subset \tilde{\omega}_{1}$ and $\omega_{1} \in U$, then $U$ is open if and only if $\left|\tilde{\omega}_{1} / U\right| \leq \omega$.

Let $\tilde{D}=\tilde{\omega}_{1} \times(\omega+1)$ and let $Y=(\omega+1) \times \omega$.

Let $\oplus$ denote disjoint topological union and let $X^{\prime}$ be the set obtained from $\tilde{D} \oplus Y$ by identifying the points $\left(\omega_{1}, n\right)$ and $(\omega, n)$ for each $n<\omega$. For each $n<\omega$, denote the point so obtained by $p_{n}$. Endow $X^{\prime}$ with the resultant quotient space topology. The desired space $X$ is obtained as a subspace of $X^{\prime}$, where $X=X^{\prime}-\left\{\left(\omega_{1}, \omega\right)\right\}$. (Since, in general, quotient maps do not preserve Tychonoff spaces, it is worthwhile to note that $X^{\prime}$ is a regular Hausdorff, Lindelöf space, and as such, $X^{\prime}$ is also a Tychonoff space.)

For each $n<\omega$, let $V_{n}$ denote the points of $X$ strictly to the right (as depicted below) of $p_{n}$. Let $A$ denote the top edge of $X$ (as depicted below) and specifically note that $X$ contains no points strictly to the right of $A$. Thus, $X$ may be depicted as

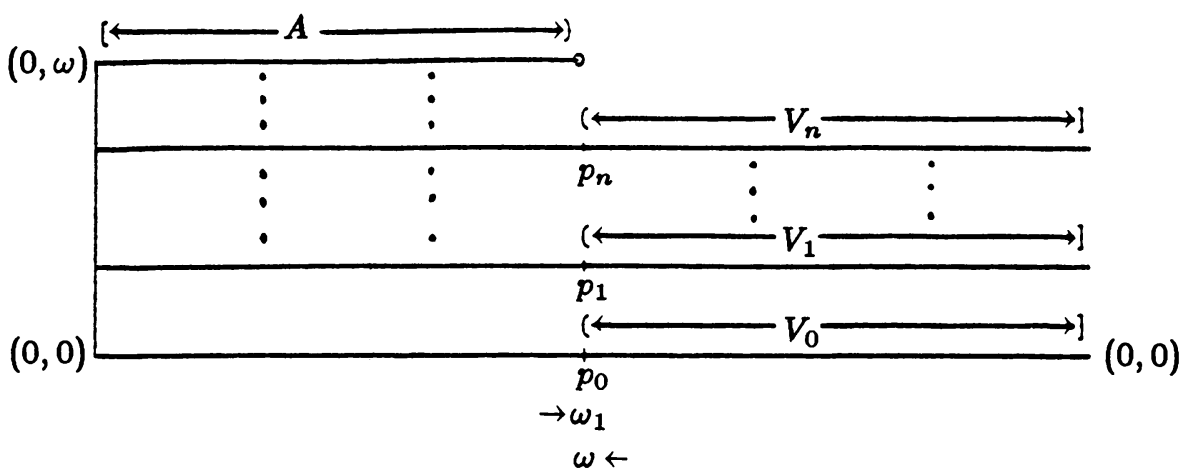

Let $V=\bigcup_{n<\omega} V_{n}$. The following assertions are easily verified.

(a) For each $n<\omega, V_{n}$ is a cozero set of $X$.

(b) The family $\left\{V_{n}\right\}_{n<\omega}$ is locally finite.

(c) For each $n<\omega, V_{n}$ and $A$ are completely separated.

(d) The sets $V$ and $A$ are not completely separated.

5.3 REMARK. The space $X$ of the preceding example is nonpseudocompact but it is not a $\sigma-\pi$ space. Furthermore, it is a nonnormal $G$-space. But $X$ possesses remote points. In fact, the much stronger condition that $T X$ is dense in $\beta X \backslash X$ 
holds $\left[\mathbf{P}_{\mathbf{3}}\right]$. The latter conclusion follows since $X$ is a nearly realcompact $[\mathbf{K}$, Theorem I, Example A], strong $G$-space $\left[\mathbf{P}_{\mathbf{2}}, \mathbf{P}_{\mathbf{3}}\right]$.

5.4 QUESTION. If $\left\{Z_{\xi}: \xi<\alpha\right\}$ is a locally finite family of zero sets of a space $X$ and if $A \subset X$ such that for each $\xi<\alpha, Z_{\xi}$ and $A$ are completely separated, then must $\bigcup_{\xi<\alpha} Z_{\xi}$ and $A$ be completely separated? (The answer is "No.", as is demonstrated by Example 5.5, below.)

5.5 EXAMPLE. The space given here is defined in [GJ, 6P]. Let

$$
\Lambda=\beta \mathbf{R} \backslash(\beta N \backslash N) \text {. }
$$

Let $A=\Lambda-\mathbf{R}$ and for each $n<\omega$, let $Z_{n}=\{n\}$. Note that $N=\bigcup_{n<\omega} Z_{n}$. The following assertions are easily verified.

(a) For each $n<\omega, Z_{n}$ is a zero set of $\Lambda$.

(b) The family $\left\{Z_{n}\right\}_{n<\omega}$ is locally finite.

(c) For each $n<\omega, Z_{n}$ and $A$ are completely separated. (In fact, for each $n<\omega$, $Z_{n}$ and $A$ are, themselves, disjoint zero sets.)

(d) The sets $N$ and $A$ are not completely separated.

ACKNOWLEDGEMENT. The second author gratefully acknowledges the mathematical stimulus of A. Dow, W. W. Comfort, S. B. Chae, A. W. Hager, G. Baloglou, R. G. Woods and the Wesleyan University Topology Seminar. This author also acknowledges, with appreciation, the financial support of Computervision Corporation.

\section{BIBLIOGRAPHY}

[CS] S. B. Chae and J. H. Smith, Remote points and G-spaces, Topology Appl. 11 (1980), 243-246. [C] W. W. Comfort, A survey of cardinal invariants, General Topology Appl. 1 (1971), 163-199.

[vD] E. K. van Douwen, Remote points, Dissertationes Math. 183 (1982).

[D] A. Dow, Remote points in large products, Topology Appl. 16 (1983), 11-17.

[FG] N. J. Fine and L. Gillman, Remote points in $\beta R$, Proc. Amer. Math. Soc. 13 (1962), 29-36. MR 26 \#732.

[G] C. L. Gates, Some structural properties of the set of remote points of a metric space, Canad. J. Math. 32 (1980), 195-209.

[GJ] L. Gillman and M. Jerison, Rings of continuous functions, Springer-Verlag, New York, 1976.

[H] M. Henriksen, personal communication, Aug. 8, 1984.

[K] A. Kato, Union of realcompact and Lindelöf spaces, Canad. J. Math. 51 (1979), 1247-1268.

[vM] J. van Mill, More on remote points, Rapport 91, Wiskundig Seminarium, Free University of Amsterdam, 1982.

$\left[\mathbf{P}_{1}\right]$ T. J. Peters, Remote points, products and G-spaces, Doctoral Dissertation, Wesleyan Univ., Middletown, CT, 1982.

$\left[\mathbf{P}_{\mathbf{2}}\right] \_, G$-spaces: Products, absolutes and remote points, Topology Proceedings 7 (1982), 119 146.

$\left[\mathbf{P}_{\mathbf{3}}\right]$ _ Dense homeomorphic subspaces of $X^{*}$ and of $(E X)^{*}$, Topology Proc. 8 (1983), 285-301.

$\left[\mathbf{P}_{\mathbf{4}}\right] \ldots$ For any $X$, the product $X \times Y$ has remote points for some $Y$, Proc. Amer. Math. Soc. 95 (1985), 641-648.

[R] S. M. Robinson, Some properties of $\beta X \backslash X$ for complete spaces, Fund. Math. 64 (1969), 335-340.

[W] R. G. Woods, Some $\aleph_{0}$-bounded subsets of Stone-Cech compactifications, Israel J. Math. 9 (1971), 250-256.

Department of Mathematics, harvey mudd College, Claremont, CaliforNIA 91711

COMPUTERVISION CORPORATION, BEDFORD, MASSACHUSETTS 01730 